PROCEEDINGS OF THE

AMERICAN MATHEMATICAL SOCIETY

Volume 127, Number 10, Pages 2905-2910

S 0002-9939(99)05083-2

Article electronically published on April 23, 1999

\title{
EXTINCTION OF SPECIES IN NONAUTONOMOUS LOTKA-VOLTERRA SYSTEMS
}

\author{
SHAIR AHMAD
}

(Communicated by Hal L. Smith)

\begin{abstract}
A nonautonomous $n$th order Lotka-Volterra system of differential equations is considered. It is shown that if the coefficients satisfy certain inequalities, then any solution with positive components at some point will have all of its last $n-1$ components tend to zero, while the first one will stabilize at a certain solution of a logistic equation.
\end{abstract}

\section{INTRODUCTION}

Consider the system of differential equations

$$
u_{i}^{\prime}(t)=u_{i}(t)\left[b_{i}(t)-\sum_{j=1}^{n} c_{i j}(t) u_{j}(t)\right], \quad c_{i j} \geq 0,
$$

$i=1, \ldots, n$. Such a system, known as the Lotka-Volterra system, models competition between $n$ species, where $u_{i}(t)$ denotes the population of the $i$ th species at time $t$. In the case where $n=2$ and the coefficients are positive numbers satisfying the inequalities

$$
\frac{b_{2}}{c_{22}}<\frac{b_{1}}{c_{12}}, \quad \frac{b_{2}}{c_{21}}<\frac{b_{1}}{c_{11}},
$$

it is well known that if $\operatorname{col}\left(u_{1}(t), u_{2}(t)\right)$ is any solution of (1.1) such that for some $t_{0}$ we have $u_{1}\left(t_{0}\right), u_{2}\left(t_{0}\right)>0$, then $u_{1}(t) \rightarrow b_{1} / c_{11}$ and $u_{2}(t) \rightarrow 0$ as $t \rightarrow \infty$. This is sometimes referred to as the principle of competitive exclusion. An extension of this principle for nonautonomous systems was given by the author in [1], where it was shown that similar algebraic inequalities imply that there can be no coexistence of the two species; one of them will be driven to extinction while the other will stabilize at a certain solution of a logistic equation. Extensions of the author's result in [1] and other similar studies have been conducted by Ahmad-Lazer [4, 5], Ahmad-Oca [3], Battauz-Zanolin [7], Ortega-Tineo [11], Redheffer [12, 13], and Oca-Zeeman [10]. Earlier, Gopalsamy [8, 9] had studied the existence and stability of periodic solutions for such systems under the assumption that the growth rates are positive and periodic, and the rest of the coefficients are positive constants. Alvarez and

Received by the editors December 15, 1997.

1991 Mathematics Subject Classification. Primary 34C35; Secondary 34D05.

Key words and phrases. Positive, component, system, extinction, exponentially.

The author wishes to acknowledge support from SISSA, Trieste, Italy, where this research was completed. 
Lazer $[6]$ extended this result to the case where all the coefficients were assumed to be positive and periodic.

In [10], Montes de Oca and Zeeman showed that if the coefficients are continuous and bounded above and below by positive constants, and if for each $i=2, \ldots, n$, there exists an integer $k_{i}<i$ such that

$$
\frac{b_{i M}}{c_{i j L}}<\frac{b_{k_{i} L}}{c_{k_{i} j M}},
$$

$j=1, \ldots, i$, then $u_{i}(t) \rightarrow 0$ exponentially for $2 \leq i \leq n$, and $u_{1}(t) \rightarrow u^{*}(t)$, where $u^{*}(t)$ is a certain solution of a logistic equation. Here, as in earlier studies, given a function $f(t), f_{M}$ and $f_{L}$ denote $\sup _{t} f(t)$ and $\inf _{t} f(t)$ respectively. For a fairly nice and detailed geometric interpretation of (1.3), the reader is referred to [10].

In this paper we consider a somewhat more general system, and give a further extension of the result in [10] by introducing a sufficient condition that is implied by the condition (1.3) stated above.

Consider the system

$$
u_{i}^{\prime}(t)=u_{i}(t)\left[b_{i}(t)+p_{i}(t)-\sum_{j=1}^{n} c_{i j}(t) u_{j}(t)\right], \quad 1 \leq i \leq n,
$$

where $c_{i j}(t)$ is continuous and bounded above and below by positive constants, $b_{i}(t)$ is continuous and $T$-periodic, $p_{i}(t)$ is continuous (not necessarily periodic) and $\left|p_{i}(t)\right| \leq c_{i} e^{-\gamma_{i} t}$, where $c_{i}$ and $\gamma_{i}$ are positive constants. We do not assume the growth rate $b_{i}(t)$ is positive; instead we assume that the average $\bar{b}_{i}=$ $\frac{1}{T} \int_{t_{0}}^{t_{0}+T} b_{i}(t) d t$ is positive. Our condition is that for each $i=2, \ldots, n$, there exist numbers $\lambda_{i 1}, \lambda_{i 2}, \ldots, \lambda_{i, i-1} \geq 0, \lambda_{i 1}+\cdots+\lambda_{i, i-1}>0$, such that

$$
\frac{\bar{b}_{i}}{c_{i j}(t)}<\frac{\lambda_{i 1} \bar{b}_{1}+\cdots+\lambda_{i, i-1} \bar{b}_{i-1}}{\lambda_{i 1} c_{1 j}(t)+\cdots+\lambda_{i, i-1} c_{i-1, j}(t)}
$$

for $j=1, \ldots, i$, and $t \geq t_{0}$ for some $t_{0}$. Then, $u_{i}(t) \rightarrow 0$ if $i \geq 2$ and $u_{1}(t) \rightarrow u^{*}(t)$, where $u^{*}$ is the unique positive solution of the logistic equation

$$
u^{\prime}(t)=u(t)\left[b_{1}(t)-c_{11}(t) u(t)\right]
$$

(see Lemma 2.1 below). It is obvious that the inequalities (1.3) imply (1.4) since one can take $\lambda_{i 1}=1$ and $\lambda_{i j}=0$ for $1<j<i$. It is easy to verify that the coefficients of the autonomous system in the following example satisfy our condition (1.4) but not their condition (1.3).

Example 1.1. Consider the system

$$
\begin{aligned}
& u_{1}^{\prime}=u_{1}\left[1-\frac{1}{2} f(t) u_{1}-\frac{1}{2} g(t) u_{2}-\frac{4}{3} h(t) u_{3}\right], \\
& u_{2}^{\prime}=u_{2}\left[1-\frac{4}{3} f(t) u_{1}-\frac{4}{3} g(t) u_{2}-\frac{1}{2} h(t) u_{3}\right], \\
& u_{3}^{\prime}=u_{3}\left[1-f(t) u_{1}(t)-g(t) u_{2}-h(t) u_{3}\right],
\end{aligned}
$$

where $f(t), g(t)$, and $h(t)$ are continuous and bounded above and below by positive constants in the interval $[1, \infty)$. It is easy to verify, by letting $\lambda_{31}=\lambda_{32}=\frac{1}{2}$ and $\lambda_{21}=1$, that this model satisfies condition (1.4). However, it does not always satisfy condition (1.3). For example, if we let $f(t)=\frac{t}{t+1}$, then (1.3) implies that either $\frac{1}{f_{L}}<\frac{1}{\frac{4}{3} f_{M}}$ or $\frac{1}{f_{L}}<\frac{1}{\frac{1}{2} f_{M}}$. Thus, either $\frac{f_{L}}{f_{M}}>\frac{4}{3}$ or $\frac{f_{L}}{f_{M}}>\frac{1}{2}$. But on $[1, \infty), \frac{f_{L}}{f_{M}}=\frac{\frac{1}{2}}{1}=\frac{1}{2}$. Therefore, condition (1.3) cannot be satisfied. 
Remark. We can further extend Example 1.1 by letting the growth rates be $b_{1}(t)=$ $\frac{1}{2}-\sin (t), b_{2}(t)=\frac{1}{2}-\cos (t)$, and $b_{3}(t)=\frac{1}{2}-\sin (t)$. The hypothesis of our theorem is still satisfied, although the growth rates are not always positive.

\section{Some PRELIMINARY RESUlts}

Throughout this paper, we assume that the functions $b_{i}(t), c_{i j}(t)$, and $p_{i}(t)$ in $(*)$ satisfy the conditions described in the introduction. We recall that $\bar{b}_{i}=$ $\frac{1}{T} \int_{t_{0}}^{t_{0}+T} b_{i}(t) d t>0,1 \leq i \leq n$, by assumption. Let $\tilde{b}_{i}(t)=b_{i}(t)-\bar{b}_{i}$, and define $B_{i}(t)=\int_{t_{0}}^{t} \tilde{b}_{i}(s) d s$. It follows that $\int_{t_{0}}^{t_{0}+T} \tilde{b}_{i}(t) d t=0$, and $B_{i}(t)$ is $T$-periodic.

Lemma 2.1. The logistic equation

$$
u^{\prime}(t)=u(t)\left[b_{1}(t)-c_{11}(t) u(t)\right]
$$

has a unique solution $u^{*}(t)$ which is bounded above and below by positive constants.

Proof. Let $P(t)=e^{B_{1}(t)}$, and let $u(t)=P(t) Y(t)$, where $B_{1}(t)=\int_{t_{0}}^{t} \tilde{b}_{1}(s) d s$. Substituting $u(t)$ in (2.1) leads to the logistic equation

$$
Y^{\prime}(t)=Y(t)\left[\bar{b}_{1}-c_{11}(t) P(t) Y(t)\right]
$$

which has a unique solution that is bounded above and below by positive constants because $\bar{b}_{1}>0$ and $c_{11} P$ is bounded above and below by positive constants (see $[1])$.

Lemma 2.2. If $\operatorname{col}\left(u_{1}(t), \ldots, u_{n}(t)\right)$ is a solution of $(*)$ such that $u_{i}\left(t_{0}\right)>0$, $1 \leq i \leq n$, for some number $t_{0}$, then there exist positive numbers $\delta$ and $\Delta$ such that $\delta \leq \sum_{i=1}^{n} u_{i}(t) \leq \Delta$ for all $t \geq t_{0}$.

Proof. Let $R_{i}(t)=\int_{t_{0}}^{t}\left(\tilde{b}_{i}(s)+p_{i}(s)\right) d s$. We note that $R_{i}(t)$ is bounded on $\left[t_{0}, \infty\right)$. Recall that $b_{i}(t)=\bar{b}_{i}+\tilde{b}_{i}(t)$. Hence letting $Q_{i}(t)=e^{R_{i}(t)}$ and making the transformation $u_{i}(t)=Q_{i}(t) X_{i}(t)$ in $(*)$ leads to the system

$$
X_{i}^{\prime}(t)=X_{i}(t)\left[\bar{b}_{i}-\sum_{j=1}^{n} c_{i j}(t) Q_{j}(t) X_{j}(t)\right]
$$

where the coefficients are bounded above and below by positive constants. The proof follows from [10] (see also [3] for an independent proof).

\section{MAin RESUlT}

Theorem 3.1. Consider the system

$$
u_{i}^{\prime}(t)=u_{i}(t)\left[b_{i}(t)+p_{i}(t)-\sum_{j=1}^{n} c_{i j}(t) u_{j}(t)\right], \quad i=1, \ldots, n .
$$

Assume that $b_{i}(t)$ is continuous T-periodic with $\bar{b}_{i}=\frac{1}{T} \int_{t_{0}}^{t_{0}+T} b_{i}(t) d t>0, p_{i}(t)$ is continuous, $\left|p_{i}(t)\right| \leq c_{i} e^{-\gamma_{i} t}$ ( $c_{i}$ and $\gamma_{i}$ positive constants), and $c_{i j}(t)$ is continuous and bounded above and below by positive constants for $1 \leq i, j \leq n$ and $t \geq t_{0}$. If condition (1.4) holds, and if $\operatorname{col}\left(u_{1}(t), \ldots, u_{n}(t)\right)$ is any solution of $(*)$ such that $u_{i}\left(t_{0}\right)>0,1 \leq i \leq n$, then $u_{i}(t) \rightarrow 0$ exponentially for $i=2, \ldots, n$, and $u_{1}(t) \rightarrow u^{*}(t)$ as $t \rightarrow \infty$, where $u^{*}(t)$ is the unique positive solution of (2.1). 
Proof. The proof is based on a subtle use of Mathematical Induction. First, we assume that the theorem holds for $n=m-1, m \geq 2$, and show that it holds for $n=m$. Let $b_{m}^{*}=\sum_{i=1}^{m-1} \lambda_{m i} \bar{b}_{i}$, and $c_{m j}^{*}(t)=\sum_{i=1}^{m-1} \lambda_{m i} c_{i j}(t)$. Our condition (1.4) for $i=m$ can now be written as

$$
\frac{\bar{b}_{m}}{c_{m j}(t)}<\frac{b_{m}^{*}}{c_{m j}^{*}(t)}, \quad 1 \leq j \leq m
$$

It is well known and easy to verify (see e.g. [1] and [5]) that the region $u_{i}(t)>0$, $1 \leq i \leq n, t \geq t_{0}$, is positively invariant. Hence, $u_{i}(t)>0$ for $t \geq t_{0}, 1 \leq i \leq n$.

Recall that $b_{i}(t)=\bar{b}_{i}+\tilde{b}_{i}(t)=\bar{b}_{i}+B_{i}^{\prime}(t)$, where $B_{i}(t)=\int_{t_{0}}^{t} \tilde{b}_{i}(s) d s$ is $T$-periodic. Thus, $(*)$ can be written as

$$
\begin{gathered}
\frac{u_{i}^{\prime}(t)}{u_{i}(t)}=\bar{b}_{i}+B_{i}^{\prime}(t)+p_{i}(t)-\sum_{j=1}^{m} c_{i j}(t) u_{j}(t), \quad 1 \leq i \leq m-1 \\
\frac{u_{m}^{\prime}(t)}{u_{m}(t)}=\bar{b}_{m}+B_{m}^{\prime}(t)+p_{m}(t)-\sum_{j=1}^{m} c_{m j}(t) u_{j}(t) .
\end{gathered}
$$

Multiplying $(* *)$ by $\lambda_{m i}$ and summing over $1 \leq i \leq m-1$, we obtain

$$
(* * * *) \quad \sum_{i=1}^{m-1} \lambda_{m i} \frac{u_{i}^{\prime}}{u_{i}}=b_{m}^{*}+\sum_{i=1}^{m-1} \lambda_{m i} B_{i}^{\prime}+\sum_{i=1}^{m-1} \lambda_{m i} p_{i}-\sum_{j=1}^{m} c_{m j}^{*} u_{j} .
$$

We note that if we let $w=\ln \left(\prod_{i=1}^{m-1} u_{i}^{\lambda_{m i}}\right)$, then $w^{\prime}=\sum_{i=1}^{m-1} \lambda_{m i} \frac{u_{i}^{\prime}}{u_{i}}$. Thus multiplying $(* * * *)$ by $\bar{b}_{m}$ and $(* * *)$ by $b_{m}^{*}$, and subtracting, we obtain

$$
\begin{aligned}
b_{m}^{*} \frac{u_{m}^{\prime}}{u_{m}}-\bar{b}_{m} w^{\prime}=\sum_{j=1}^{m} & \left(\bar{b}_{m} c_{m j}^{*}-b_{m}^{*} c_{m j}\right) u_{j}-\sum_{i=1}^{m-1} \lambda_{m i} \bar{b}_{m} B_{i}^{\prime} \\
& -\sum_{i=1}^{m-1} \lambda_{m i} \bar{b}_{m} p_{i}+b_{m}^{*} p_{m}+b_{m}^{*} B_{m}^{\prime} .
\end{aligned}
$$

Therefore

$$
b_{m}^{*} \frac{u_{m}^{\prime}}{u_{m}}-\bar{b}_{m} w^{\prime} \leq \sum_{j=1}^{m}\left(\bar{b}_{m} c_{m j}^{*}(t)-b_{m}^{*} c_{m j}(t)\right) u_{j}(t)+\Gamma(t)
$$

where $\Gamma(t)=b_{m}^{*} B_{m}^{\prime}+b_{m}^{*} p_{m}-\sum_{i=1}^{m-1} \lambda_{m_{i}} \bar{b}_{m} p_{i}-\sum_{i=1}^{m-1} \lambda_{m i} \bar{b}_{m} B_{i}^{\prime}$. Recall that for each $i, 1 \leq i \leq n, B_{i}(t)=\int_{t_{0}}^{t} \bar{b}_{i}(s) d s$ is $T$-periodic and hence bounded. Also for each $i, 1 \leq i \leq n,\left|p_{i}(t)\right| \leq c_{i} e^{-\gamma_{i} t}$. Therefore, we can assume that there exists a positive number $M$ such that $\int_{t_{0}}^{t} \Gamma(t) d t \leq M$. In view of (3.1), there exists a number $\alpha>0$ such that

$$
\bar{b}_{m} c_{m j}^{*}(t)-b_{m}^{*} c_{m j}(t)=c_{m j}^{*}(t) c_{m j}(t)\left[\frac{\bar{b}_{m}}{c_{m j}(t)}-\frac{b_{m}^{*}}{c_{m j}^{*}(t)}\right]<-\alpha,
$$

$1 \leq j \leq m$. In view of Lemma 2.2, if we let $v=\prod_{i=1}^{m-1} u_{i}^{\lambda_{m i}}$ so that $w=\ln v$, then for a suitable constant $\beta>0$, one can write

$$
\frac{d}{d t} \ln \frac{u_{m}^{b_{m}^{*}}}{v^{\bar{b}_{m}}}=b_{m}^{*} \frac{u_{m}^{\prime}}{u_{m}}-\bar{b}_{m} w^{\prime}<-\beta+\Gamma(t)
$$


Integrating both sides, we obtain

$$
\ln \frac{u_{m}(t)^{b_{m}^{*}}}{v(t)^{\bar{b}_{m}}} \leq-\beta\left(t-t_{0}\right)+k, \quad k \text { a constant. }
$$

Therefore,

$$
u_{m}(t)^{b_{m}^{*}} \leq\left(\prod_{i=1}^{m-1} u_{i}^{\lambda_{m i}}\right)^{\bar{b}_{m}} \cdot e^{k} \cdot e^{-\beta\left(t-t_{0}\right)}
$$

Since $0<u_{i}(t)<\Delta$ for $t \geq t_{0}, 1 \leq i \leq m-1$, it follows that $u_{m}(t) \rightarrow 0$, exponentially, as $t \rightarrow \infty$.

Next, we need to show that $u_{i}(t) \rightarrow 0$ exponentially for $i=2, \ldots, m-1$. We accomplish this by rewriting the system $(*)$ as

$$
u_{i}^{\prime}(t)=u_{i}(t)\left[b_{i}(t)+p_{i}^{*}(t)-\sum_{j=1}^{m-1} c_{i j}(t) u_{j}(t)\right]
$$

$i=1,2, \ldots, m-1$, and $p_{i}^{*}(t)=p_{i}(t)-c_{i m}(t) u_{m}(t)$. It follows that $p_{i}^{*}(t)$ satisfies the hypothesis of our theorem since $p_{i}(t)$ and $u_{m}(t)$ both tend to zero exponentially, and $c_{i m}(t)$ is bounded above and below by positive constants. We note that the inequalities in (1.4) are independent of the $n$th equation in the sense that by dropping the $n$th case, the coefficients of the smaller system $\left(*^{\prime}\right)$ still satisfy inequalities (1.4). Hence, applying the induction hypothesis to the smaller system $\left(*^{\prime}\right)$, it follows that for $2 \leq i \leq m-1, u_{i}(t) \rightarrow 0$ as $t \rightarrow \infty$.

Now, we need to show that the theorem holds for $n=1$. For $n=1$, the system $(*)$ reduces to the logistic equation

$$
u_{1}^{\prime}(t)=u_{1}(t)\left[b_{1}(t)+p_{1}(t)-c_{11} u_{1}(t)\right] .
$$

Hence, we need to show that $u_{1}(t)-u^{*}(t) \rightarrow 0$, where

$$
u^{* \prime}(t)=u^{*}(t)\left[b_{1}(t)-c_{11} u^{*}(t)\right]
$$

as described in Lemma 2.1.

Let $\sigma(t)=\left|\ln \frac{u_{1}(t)}{u^{*}(t)}\right|$. Then,

$$
\begin{aligned}
\sigma^{\prime}(t) & =\operatorname{sgn}\left(u_{1}(t)-u^{*}(t)\right)\left[p_{1}(t)-c_{11}(t) u_{1}(t)+c_{11}(t) u^{*}(t)\right] \quad \text { a.e. } \\
& =\hat{p}_{1}(t)-c_{11}(t)\left|u_{1}(t)-u^{*}(t)\right| \quad \text { a.e. }
\end{aligned}
$$

where $\hat{p}_{1}(t)=\operatorname{sgn}\left(u_{1}(t)-u^{*}(t)\right) p_{1}(t)$. Integrating both sides, we obtain

$$
\sigma(t)=\sigma\left(t_{0}\right)+\int_{t_{0}}^{t} \hat{p}_{1}(s) d s-\int_{t_{0}}^{t} c_{11}(s)\left|u_{1}(s)-u^{*}(s)\right| d s .
$$

It follows from Lemmas 2.1 and 2.2 that $\sigma(t)=\left|\ln \frac{u_{1}(t)}{u^{*}(t)}\right|$ is bounded. Hence, we have $\int_{t_{0}}^{t}\left|u_{1}(s)-u^{*}(s)\right| d s<\infty$. This, along with the fact that $u_{1}^{\prime}(t)-u^{* \prime}(t)$ is bounded, implies that $u_{1}(t)-u^{*}(t) \rightarrow 0$ as $t \rightarrow \infty$. The proof of the theorem is now complete. 


\section{REFERENCES}

[1] S. Ahmad, On the nonautonomous Volterra-Lotka competition equations, Proc. Amer. Math. Soc. 117 (1993), 199-204. MR 93c:34109

[2] _ Convergence and ultimate bounds of solutions of the nonautonomous Volterra-Lotka competition equations, J. Math. Anal. Appl. 127 (1987), 377-387. MR 89a:92032

[3] S. Ahmad and F. Montes de Oca, Extinction in nonautonomous T-periodic competitive LotkaVolterra systems, Appl. Math. Comput. 90 (1998), 155-166. MR 98h:92031

[4] S. Ahmad and A. C. Lazer, On the nonautonomous N-competing species problems, Applicable Anal. 57 (1995), 209-323. MR 97a:34128

[5] — Necessary and sufficient average growth in a Lotka-Volterra system, to appear in Nonlinear Analysis.

[6] C. Alvarez and A. C. Lazer, An application of topological degree to the periodic competing species problem, J. Austral. Math. Soc. Ser. B 28 (1986), 202-219. MR 87k:34062

[7] A. Battauz and F. Zanolin, Coexistence states for periodic competitive Kolmogorov systems, J. Math. Anal. Appl. 219 (1998), 179-199. CMP 98:09

[8] K. Gopalsamy, Exchange of equilibria in two species Lotka-Volterra competition models, J. Austral. Math. Soc. Ser. B 24 (1982), 160-170. MR 83k:34034

[9] _ Global asymptotic stability in a periodic Lotka-Volterra system, J. Austral. Math. Soc. Ser. B 27 (1985), 66-72. MR 86f:34094

[10] F. Montes de Oca and M. L. Zeeman, Extinction in nonautonomous competitive LotkaVolterra systems, Proc. Amer. Math. Soc. 124 (1996), 3677-3687. MR 97b:92017

[11] R. Ortega and A. Tineo, An exclusion principle for periodic competitive systems in three dimensions, Nonlinear Anal. 31 (1998), 883-893. MR 98j:34049

[12] R. Redheffer, Nonautonomous Lotka-Volterra systems, I, J. Diff. Eq. 127 (1996), 519-541. MR 97f:34023

[13] R. Redheffer, Nonautonomous Lotka-Volterra systems, II, J. Diff. Eq. 132 (1996), 1-20. MR 98d:34071

[14] A. Tineo and C. Alvarez, A different consideration about the globally asymptotically stable solution of the periodic n-competing species problem, J. Math. Anal. Appl. 159 (1991), 44-50. MR 93d:34080

Division of Mathematics and Statistics, The University of Texas at San Antonio, San Antonio, Texas 78249

E-mail address: ahmad@sphere.math.utsa.edu 